\title{
Plasma impurities observed by a pulse height analysis diagnostic during the divertor campaign of the Wendelstein 7-X stellarator a)
} \author{
R. Burhenn, ${ }^{2}$ B. Buttenschön, ${ }^{2}$ B. Geiger, ${ }^{2}$ O. Grulke, ${ }^{2}$ A. Langenberg, ${ }^{2}$ O. Marchuk, ${ }^{3}$ \\ and the W7-X team ${ }^{\mathrm{c}}$ \\ ${ }^{I}$ Institute of Plasma Physics and Laser Microfusion, Hery 23, 01-497 Warsaw, Poland \\ ${ }^{2}$ Max Planck Institute for Plasma Physics, 17491 Greifswald, Germany \\ ${ }^{3}$ Forschungszentrum 52425 Juelich, Germany \\ ${ }^{4}$ Laboratorio Nacional de Fusion, CIEMAT, Avenida Complutense, Madrid, Spain \\ ${ }^{5}$ Princeton Plasma Physics Laboratory, Princeton, NJ, USA
}

M. Kubkowska, ${ }^{1, \text { b) }}$ A. Czarnecka, ${ }^{1}$ T. Fornal, ${ }^{1}$ M. Gruca, ${ }^{1}$ S. Jabłonski, ${ }^{1}$ N. Krawczyk, ${ }^{1}$ L. Ryć, ${ }^{1}$ K. J. McCarthy, ${ }^{4}$ U. Neuner, ${ }^{2}$ D. Nicolai, ${ }^{3}$ N. Pablant, ${ }^{5}$ B. Schweer, ${ }^{3}$ H. Thomsen, ${ }^{2}$ Th. Wegner, ${ }^{2}$

(Presented XXXXX; received XXXXX; accepted XXXXX; published online XXXXX)

The paper reports on the optimization process of the soft X-ray pulse height analyzer (PHA) installed on the Wendelstein 7-X (W7-X) stellarator. It is a 3-channel system that records X-ray spectra in the range from 0.6 to $19.6 \mathrm{keV}$. X-ray spectra, with temporal and spatial resolution of $100 \mathrm{~ms}$ and $2.5 \mathrm{~cm}$ (depending on selected slit sizes), respectively, are line integrated along a line-of-sight that crosses near to the plasma center. In the second W7-X operation phase with a carbon test divertor unit, light impurities, e.g., carbon and oxygen, were observed as well as mid- to high-Z elements, e.g., sulfur, chlorine, chromium, manganese, iron and nickel. In addition, X-ray lines from several tracer elements have been observed after laser blow-off injection of different impurities, e.g., silicon, titanium and iron, and during discharges with prefill or gas puff of neon or argon. These measurements were achieved by optimizing light absorber-foil selection, which defines the detected energy range, and remotely controlled pinhole size, which defines photon flux. The identification of X-ray lines was confirmed by other spectroscopic diagnostics, e.g., by the High-Efficiency XUV Overview Spectrometer, HEXOS and high-resolution X-ray imaging spectrometer, HR-XIS.

\section{INTRODUCTION}

In many fusion devices, soft X-ray (SXR) diagnostics are critical systems which deliver information about plasma parameters, like electron $\left(T_{e}\right)$ and ion temperature $\left(T_{i}\right)$ or impurities density $\left(n_{i}\right)$. The collected radiation is also suitable for performing other plasma processes such as impurity transport studies. In particular, a pulse height analysis (PHA) system is a common diagnostic used in many tokamaks and stellarators ${ }^{1-4}$ for identification of plasma impurities, $T_{e}$ estimation $^{5-6}$, study of non-thermal electrons (if present) ${ }^{7}$ and estimation of $Z_{\text {eff }}$ (by comparison of experimental with modeled spectra ${ }^{8}$. Such a system has been installed on the Wendelstein 7-X stellarator ${ }^{9-10}$ which was commissioned at the end of 2015 and in 2016 had its first experimental campaign ${ }^{11-12}$. In 2017, after the installation of 10 uncooled divertor modules and about 8000 graphite wall tiles, the first part of its second experimental campaign took place. The PHA system for $\mathrm{W} 7-\mathrm{X}$ was commissioned and tested during the first operational phase, OP1.1 $1^{13-14}$, while the optimization of the diagnostic was done during this second operational phase, OP1.2a. The

\footnotetext{
a) Published as part of the Proceedings of the 22nd Topical Conference on High-Temperature Plasma Diagnostics (HTPD 2018) in San Diego, California, USA.

b) corresponding author: monika.kubkowska@ipplm.pl

c) R.C. Wolf et al. Nuclear Fusion 57, 102020 (2017).
}

diagnostic consists of 3 independent channels which are dedicated to measuring SXR spectra in different ranges to detect and follow the evolution of low-Z (like carbon, oxygen, nitrogen), mid-Z (like sulphur, chlorine, argon) and high-Z impurities (like chromium, iron, nickel, copper). These measurements were possible through the use of Silicon Drift Detectors (SDD), one covered by a thin Polymer window, and the other two by $8 \mu \mathrm{m}$ thick beryllium foils plus an additional filter interchange system. Through appropriate settings, the PHA system at $\mathrm{W} 7-\mathrm{X}$ is distinguished by a quite high energy resolution $(150 \mathrm{eV}$ FWHM at $5.9 \mathrm{keV}$ ) thereby making the system suitable for impurity transport studies ${ }^{15}$.

This paper firstly describes briefly the experimental setup and optimized settings of the PHA system. Secondly, it describes the energy and intensity calibration of collected spectra as well as the impact of the pileup effect. The next section presents representative PHA results obtained during the OP1.2a experimental campaign for comparison with High-Efficiency XUV Overview Spectrometer (HEXOS) and High-Resolution X-ray Imaging Spectrometer (HRXIS) diagnostics. The paper is completed by a short summary of the optimization process of the PHA system. 


\section{SETTINGS OF THE PHA SYSTEM DURING W7-X EXPERIMANTAL CAMPAIGN OP1.2A}

The W7-X PHA spectrometer is designed to acquire spectra in the energy range from 0.6 to $19.6 \mathrm{keV}$ (assuming $1 / \mathrm{e}$ transmission) in 3 energy channels. The measured X-ray plasma emission consists of a continuum spectrum, which diminishes considerably with energy, plus spectral line components. To have the possibility of obtaining higher statistics and revealing details of the spectrum at higher energy where intensity is low, the PHA system for W7-X is designed to acquire spectra in three ranges: $1.55-19.60 \mathrm{keV}$, $0.96-19.60 \mathrm{keV}$ and $0.6-19.60 \mathrm{keV}$, these corresponding to its $1^{\text {st }}, 2^{\text {nd }}$ and $3^{\text {rd }}$ PHA channel, respectively. It is performed by the use of three SDDs covered by different filters located behind and a set of adjustable slits (square shape) which regulates the flux of radiation that reaches the detectors. The quality of the acquired spectra is determined by sufficient counting statistics in the individual channels. This was achived by setting an optimized acquisition time and slit widths. In OP1.2a the acquisition time was chosen to be 100 $\mathrm{ms}$. This choice is a compromise between the quality of collected spectra (determined by statistics) and the temporal resolution required for the diagnostics.

The presented PHA system works in the Multi-Channel Analyzer (MCA) mode where the user defines the bin width and corresponding MCA channel number. The primary spectrometer settings are, among other, the MCA bin width and the number of bins. Together, these settings determine the energy range of the MCA. The spectrum ranges from zero to a maximum energy equal to the number of spectrum bins multiplied by the MCA bin width. During OP1.2a, in order to obtain sufficiently good energy resolution as well as the granularity of the acquired spectra, the following values of the bin widths were set: $30 \mathrm{eV}, 30 \mathrm{eV}$ and $20 \mathrm{eV}$ for the $1^{\text {st }}, 2^{\text {nd }}$ and $3^{\text {rd }}$ PHA channel, respectively. Nominally, the MCA channels number ranges between 256 and 16384. In the case of the PHA system it was set between 256 and 1024 depending on the observed energy range. For efficient operation of the spectrometer and, in consequence, good quality spectra, the total radiation from a plasma (expressed in terms of input count rate, ICR) reaching the detectors should vary within a certain range. In general, the slit widths were set in accordance with the plasma heating power and energy. Therefore, the slit widths were starting from $25 \mu \mathrm{m}$ for the $3^{\text {rd }}$ channel (without additional beryllium foil, designed for measurement of light impurities) to 300 and $800 \mu \mathrm{m}$ for the $2^{\text {nd }}$ and $1^{\text {st }}$ PHA channel, which are intended for measurements medium and high-Z impurities.

\section{CALIBRATION OF THE PHA SPECTRA}

The PHA spectra are energy calibrated by the use of fluorescence spectra ${ }^{16}$. A mini X-ray tube (from Amptek ${ }^{17}$ ) with a silver anode is used as an excitation source for target materials (stainless steel and $\mathrm{Cu}$ plates) located inside its vacuum chamber. Based on the collected signals which consist of 8-10 (depending on settings - MCA channel number and width) known fluorescence lines, energy calibration of the PHA detectors is made for all 3 channels independently. An example of a collected spectrum is presented in Fig 1.

To study impurity behavior in W7-X discharges, intensity calibration of the PHA spectra is also needed. For this aim, the diagnostic geometry, the influence of filters, the detector efficiency and the efficiency of electronics (dead time) must be considered. Figure 2 shows a schematic view of plasma observation by the PHA system with the viewing cone defined by the position of detector and slits. In this present consideration, plasma within the viewing cone is divided into many small points with index $i$.

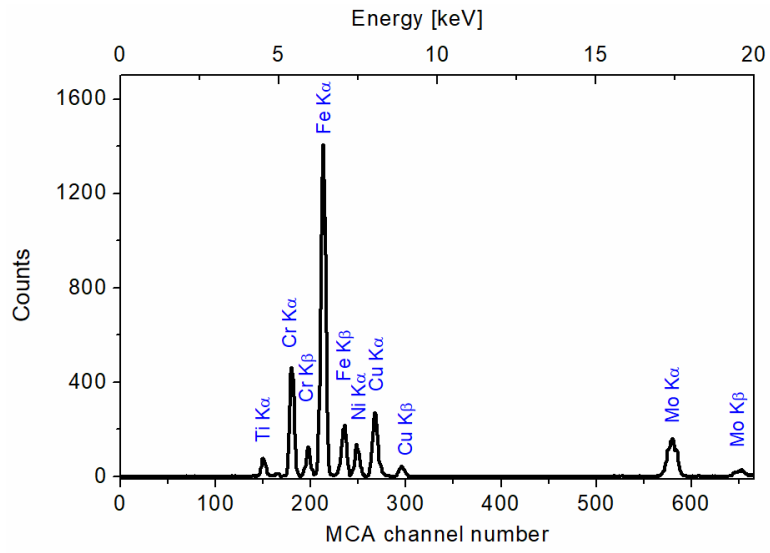

FIG.1.Fluorescence spectrum used for energy calibration of the PHA system installed at W7-X. The spectrum was collected for the following PHA operational settings: $t_{p}=1 \mu \mathrm{s}, \mathrm{MCA}$ bin width $=30 \mathrm{eV}$, $\mathrm{MC}$ channel number $=1024$.

It should be noted here that the X-ray sensitive area of the detectors is circular in shape while the pinholes are square, so a cone shaped viewing volume is an approximation. Thus, each plasma radiating element has volume $\Delta V_{i}$ that is positioned at a distance of $R_{i}$ from the detector. The detector surface is described by the parameter $S_{D}$. Hence, the number of photons $N_{i}$ of energy $E_{j}$ emitted by the point $i$ inside the solid angle in the time period $\Delta t$ (in the case of W7-X PHA system in $100 \mathrm{~ms}$ - PHA acquisition time), can be calculated as follows:

$N_{i}\left(E_{j}\right)=\varepsilon_{i}\left(E_{j}\right) \cdot \Delta V_{i} \cdot \Delta t \cdot \frac{\Delta E}{E_{j}}$

where $\varepsilon_{i}$ is plasma emissivity in $\left[\mathrm{W} / \mathrm{m}^{3} / \mathrm{eV}\right]$.

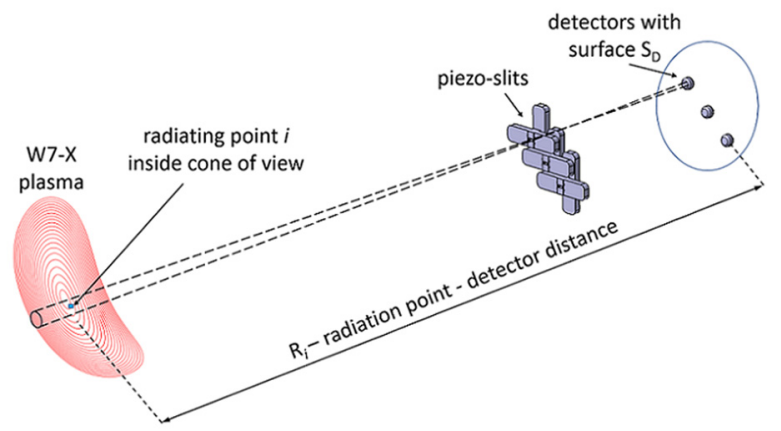

FIG. 2. A scheme showing the observation geometry of the plasma by a single PHA channel.

Next, by taking into account, that the $\mathrm{X}$-ray radiation is isotropic and that the detector surface is located at the 
distance of $R_{i}$ from the radiating point $i$, the integrated number of photons $N^{D}$ that reach the detector surface, $S_{D}$, (origin from the plasma volume inside the cone of view) can be described by:

$$
N^{D}\left(E_{j}\right)=\sum_{i} N_{i}\left(E_{j}\right) \cdot \frac{S_{D}}{4 \pi R_{i}^{2}}
$$

Finally, the average emissivity as a function of the collected number of photons can be described as follows:

$\left\langle\varepsilon\left(E_{j}\right)\right\rangle=\frac{N^{D}\left(E_{j}\right) \cdot E_{j}}{\Delta t \cdot \Delta E} \cdot A \cdot M$

where $A$ is a geometrical parameter which accounts for the fact that the photons reach a detector surface under different angles $\alpha_{i}$,

$$
A=\frac{4 \pi}{S_{D} \sum_{i} \frac{\Delta V_{i}}{R_{i}^{2}} \cos \alpha_{i}}
$$

while $M$ is a correction factor which includes the pile-up effect that can give rise to the loss of counts.

This factor is calculated assuming that the output count rate (OCR) is related to the input count rate (ICR) by the following formula:

$$
O C R=I C R \cdot \exp \left(-I C R \cdot 2\left(t_{p}+t_{g}\right)\right)
$$

where $t_{p}$ and $t_{g}$ are adjustable parameters of the digital signal processor (DSP), called peaking and gap times, respectively. These parameters define the shape of the trapezoidal digital filter used in the DSP.

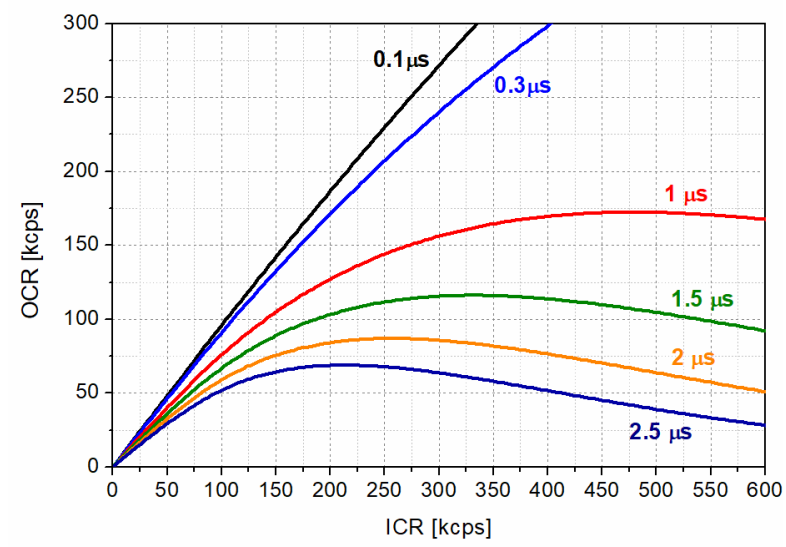

FIG.3.Output Count Rate versus Incident Count Rate for gap time $t_{g}$ of $60 \mathrm{~ns}$ and various peaking times, $t_{p}$.

Figure 3 presents number of measured photons versus number of incoming counts for several $t_{p}$. It should be noted that, for the ICR range plotted here, there is a possibility to have the same value of OCR for two different values of ICR. To adjust for a high OCR (close to the curve maximum) it is necessary to adjust the slits of variable width which can regulate photon flux. It is worth adding that $t_{p}$ also has impacts on the energy resolution of collected spectra. For this reason, a compromise needs to be found to observe good resolution and good quality W7-X spectra (with good statistics - high enough level of counts (photons) to resolve the spectra and identify impurity lines).

\section{EXPERIMENTAL RESULTS}

During the first W7-X test divertor campaign OP1.2a, the PHA system was set to observe spectra which include spectral lines of low- and high- $\mathrm{Z}$ plasma impurities. Figure 4 presents detector response curves for the 3 PHA channels on which the energies of the principal expected impurity line positions have been overlaid.

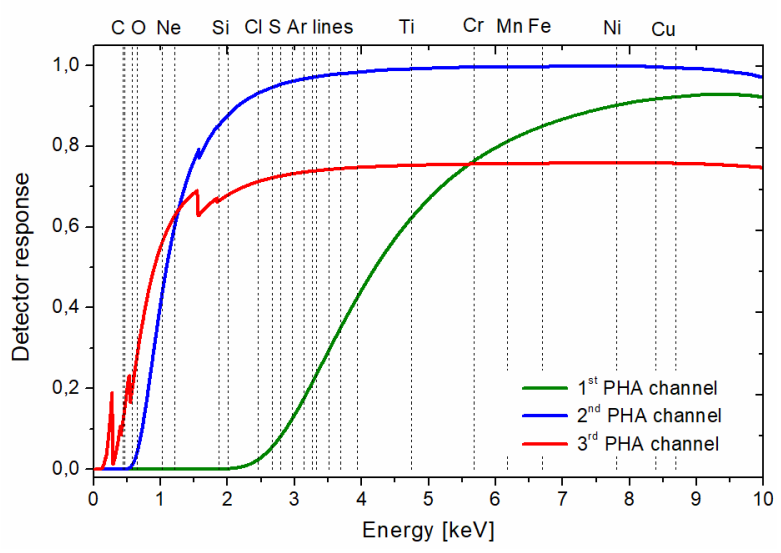

FIG.4.Detector response curves for the 3 PHA channels using during the OP1.2a W7-X experimental campaign. The relative positions of expected resonant He-like and/or H-like plasma impurity lines are also indicated.

Next, Fig. 5 shows examples of spectra collected by the 3 channels. These have been accumulated along a full discharge.

TABLE I. List of impurity lines identified in W7-X plasmas using the PHA system. Underlined lines

\begin{tabular}{|c|c|c|}
\hline Type of line & Energy [keV] & Wavelength [nm] \\
\hline $\mathrm{H}$-like $\mathrm{C}\left(\mathrm{C}^{+5}\right)$ & 0.436 & 2.847 \\
\hline $\mathrm{H}$-like $\mathrm{C}\left(\mathrm{C}^{+5}\right)$ & 0.459 & 2.699 \\
\hline He-like $O\left(\mathrm{O}^{+6}\right)$ & 0.574 & 2.160 \\
\hline H-like $\mathrm{O}\left(\mathrm{O}^{+7}\right)$ & 0.653 & 1.898 \\
\hline He-like $\mathrm{Ne}\left(\mathrm{Ne}^{+8}\right)$ & 0.922 & 1.345 \\
\hline $\mathrm{H}$-like $\mathrm{Ne}\left(\mathrm{Ne}^{+9}\right)^{\prime}$ & 1.022 & 1.213 \\
\hline He-like Si $\left(\mathrm{Si}^{+12}\right)$ & 1.865 & 0.665 \\
\hline H-like Si $\left(\mathrm{Si}^{+13}\right)$ & 2.005 & 0.618 \\
\hline$\overline{\text { He-like S }\left(\mathrm{S}^{+14}\right)}$ & 2.460 & 0.504 \\
\hline $\mathrm{H}$-like $\mathrm{S}\left(\mathrm{S}^{+15}\right)$ & 2.662 & 0.466 \\
\hline He-like $\mathrm{Cl}\left(\mathrm{Cl}^{+15}\right)$ & 2.789 & 0.444 \\
\hline H-like $\mathrm{Cl}\left(\mathrm{Cl}^{+16}\right)$ & 2.961 & 0.419 \\
\hline He-like $\operatorname{Ar}\left(\mathrm{Ar}^{+16}\right)$ & 3.140 & 0.395 \\
\hline He-like $\mathrm{Cl}\left(\mathrm{Cl}^{+15}\right)$ & 3.267 & 0.379 \\
\hline $\mathrm{H}$-like $\operatorname{Ar}\left(\mathrm{Ar}^{+17}\right)$ & 3.321 & 0.373 \\
\hline $\mathrm{H}$-like $\mathrm{Cl}\left(\mathrm{Cl}^{+16}\right)$ & 3.508 & 0.353 \\
\hline He-like $\operatorname{Ar}\left(\mathrm{Ar}^{+16}\right)$ & 3. 684 & 0.336 \\
\hline H-like $\operatorname{Ar}\left(\mathrm{Ar}^{+17}\right)$ & 3.935 & 0.315 \\
\hline He-like $\mathrm{Ti}\left(\mathrm{Ti}^{+20}\right)$ & 4.749 & 0.261 \\
\hline He-like $\mathrm{Cr}\left(\mathrm{Cr}^{+22}\right)$ & 5.682 & 0.218 \\
\hline He-like $\mathrm{Mn}\left(\mathrm{Mn}^{+23}\right)$ & 6.180 & 0.201 \\
\hline He-like $\mathrm{Fe}\left(\mathrm{Fe}^{+24^{*}}\right)$ & 6.700 & 0.185 \\
\hline He-like $\mathrm{Ni}\left(\mathrm{Ni}^{+26}\right)$ & 7.805 & 0.159 \\
\hline He-like $\mathrm{Cu}\left(\mathrm{Cu}^{+27}\right)$ & 8.391 & 0.015 \\
\hline
\end{tabular}
correspond to impurities injected during laser blow-off (LBO) experiments.

*this line was observed also in discharges without LBO

In Table I, all identified spectral lines during first W7$\mathrm{X}$ divertor campaign have been listed. These correspond to $\mathrm{He}$ - and/or H-like ions of intrinsic (carbon, oxygen, sulphur, chlorine, chromium, manganese, iron, nickel, copper) or injected elements (neon, silicon, argon, titanium as well as iron). The correct optimization of all PHA channels during 
OP1.2a enabled first time observation of the higher $\mathrm{Z}$ elements such as $\mathrm{Cr}, \mathrm{Mn}, \mathrm{Fe}, \mathrm{Ni}$ and $\mathrm{Cu}$ which were not observed during the previous OP1.1 limiter campaign ${ }^{13}$.

In the case of carbon lines, the energy resolution of the PHA system for the applied settings, was unfortunately too poor to resolve CVI lines at $435.5 \mathrm{eV}$ and at $459.4 \mathrm{eV}$. Nonetheless an intense peak is present at these energies and is associated to the lines.
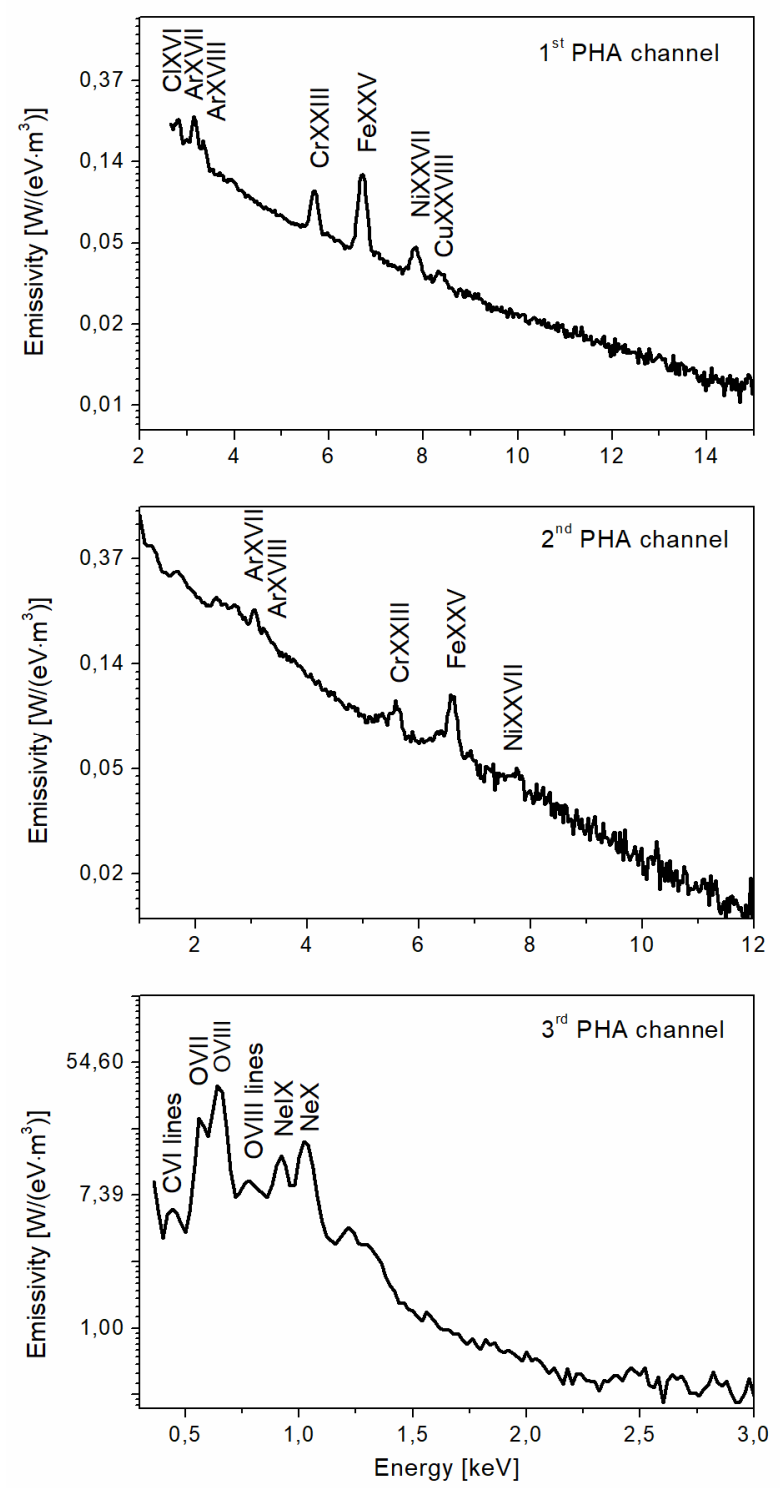

FIG.5. An example of collected by 3 PHA channels spectra for discharge \#20171129.021 during OP1.2a experimental campaign at W7-X. Identified spectral lines are highlighted.

The presence in the W7-X plasma of all the impurities, identified in Table I, has been confirmed by other spectroscopy based diagnostics, e.g. by the High-Efficiency XUV Overview Spectrometer HEXOS ${ }^{18-19}$. A spectrum obtained by HEXOS for a discharge created during the same experiment series is presented in figure 6 for comparison.

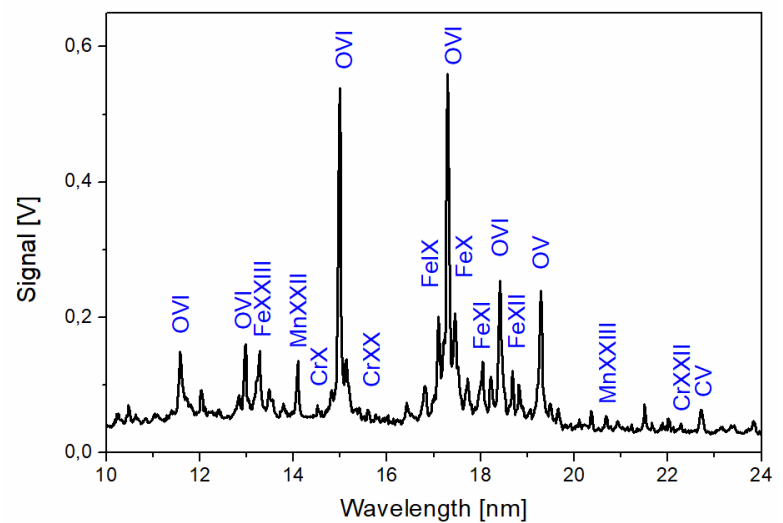

FIG.6. An example of a spectrum for discharge 20171129.025 with identified lines observed by the HEXOS spectrometer. Some lines are still to be identified. The spectrum is composed of peak signal levels per pixel over the whole discharge.

Several X-ray lines observed by the PHA system were also observed by the X-ray imaging crystal spectrometer systems $^{20-21}$ XICS and HR-XIS (high resolution). These diagnostics are equipped with crystals suited to measurements of He- and $\mathrm{H}$-like Ar lines, as well as lines from high-Z impurities, i.e. Fe XXV, Mo XXXIII, Si XIII, Ti XXI, Ni XXVII, Cu XXVIII and W LXV with high temporal resolution $(\sim 2 \mathrm{~ms})$.

In the examples shown here the PHA system has 100 ms time resolution. However, it should be noted that line intensity changes during the discharge are also observed. Figure 7 presents time traces, after the injection of tracer elements by laser blow-off ${ }^{21}$, collected by the PHA and HRXICS systems. It is seen that the signals from both diagnostics are well correlated and despite the lower PHA time resolution in comparison with the HR-XIS system, the decay time of injected impurities, which corresponds to the transport time ${ }^{22}$, can be observed. In the case of Fe injection (figure 7c), a small background level of Fe XXV line is still observed in the PHA signal after the decay time of injected $\mathrm{Fe}$ in HR-XIS signal. The explanation of this observation requires detailed analysis of collected spectra, especially that the Fe is also a W7-X wall material. 

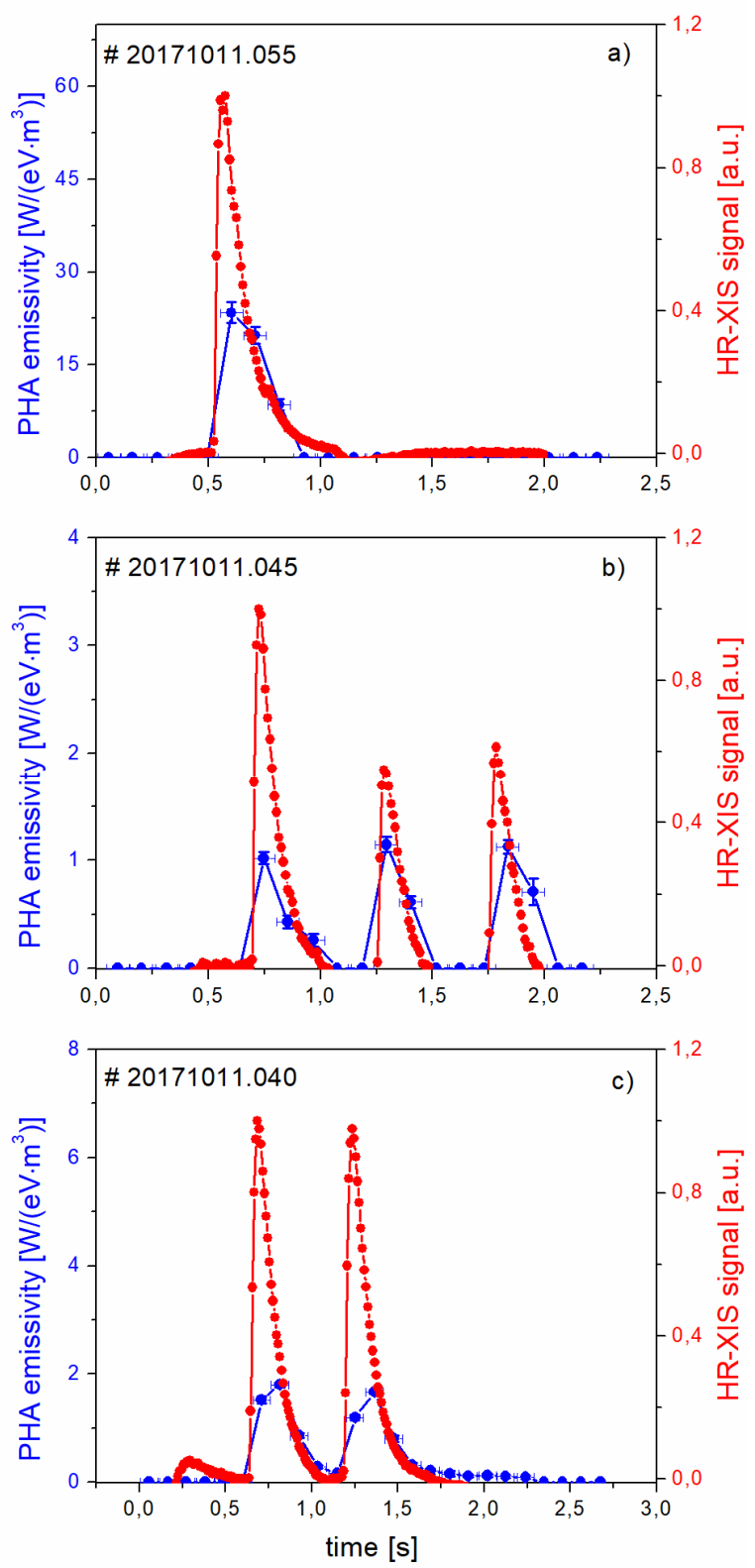

FIG.7. Comparison of time traces of Si XIII (a), Ti XXI (b) and Fe XXV (c) lines during the laser blow-off injection. - - - corresponds to PHA signal and ——- corresponds to HR-XIS signal.

\section{SUMMARY}

During the operational phase OP1.2a of W7-X, 3 PHA channels have been optimized to deliver spectra with high enough level of photon numbers to identify plasma impurities over a broad X-ray energy range. This was possible by proper selection of absorber-foil, which defines the beginning of detected energy range; remotely controlled pinhole size, which defines photon flux number; and proper settings of MCA parameters. Identification of plasma impurities from low to higher Z-elements was possible by choosing different energy ranges for each of the 3 PHA channels which gave in total energy range from about $600 \mathrm{eV}$ up to $19.6 \mathrm{keV}$ (assuming 1/exp transmission). In the paper, the procedure used for spectral calibration has been presented including treatment of the pile-up effect which was at the level of $10 \%$ during OP1.2a. Identified impurities include those originating from vacuum chamber wall material of $\mathrm{W} 7-\mathrm{X}(\mathrm{C}, \mathrm{S}, \mathrm{Cr}, \mathrm{Mn}, \mathrm{Fe}, \mathrm{Ni}$ and $\mathrm{Cu})$ and those from dedicated tracer injection experiments $(\mathrm{Si}, \mathrm{Ti}$, $\mathrm{Ne}, \mathrm{Ar})$. Also, $\mathrm{Cl}$, which probably originated from some PVC materials located in the W7-X chamber, is still observed (also seen during OP1.1). However, its line intensity is lower than during the first experimental campaign (comparing discharges in similar conditions) which could suggest lower content of this impurity in the W7-X plasma. In the next step, it is planned to test higher $t_{p}$ values, especially in the case of $3^{\text {rd }}$ PHA channel to try to increase the energy resolution and resolve carbon lines. Nevertheless, the PHA system at W7-X is optimized and ready for the second part of divertor operation to study impurity behavior variations during the discharges.

\section{ACKNOWLEDGMENTS}

This work has been carried out within the framework of the EUROfusion Consortium and has received funding from the Euratom research and training programme 2014-2018 under grant agreement No 633053. The views and opinions expressed herein do not necessarily reflect those of the European Commission.

This scientific work was partly supported by Polish Ministry of Science and Higher Education within the framework of the scientific financial resources in the years 2014-2018 allocated for the realization of the international co-financed project.

\section{REFERENCES AND FOOTNOTES}

${ }^{1}$ K. W. Hill et al., Rev. Sci. Instrum. 56, 840 (1985).

${ }^{2}$ S. Muto et al., Rev. Sci. Instrum. 27, 1206 (2001).

${ }^{3}$ Ch. Zhongyong et al., Plasma Science \& Technology 7(3), 2813 (2005).

${ }^{4}$ T.I..Madeira et al., Proceedings of the 29th EPS Conference on Controlled Fusion and Plasma Physics, Montreux, 17-21 June 2002 (unpublished) Vol. 26B, P-4.122.

${ }^{5}$ A. Weller et al., Proceedings of the 38th EPS Conference on Controlled Fusion and Plasma Physics, 2011 (unpublished) P5.054.

${ }^{6} \mathrm{~N}$. Krawczyk et al., Fus. Eng. Design https://doi.org/10.1016/j.fusengdes.2018.04.120.

${ }^{7}$ J. E. Rice et al., Phys Rev. A25, 1645 (1982).

${ }^{8}$ D. Pasini et al., Rev. Sci. Instrum. 59, 693 (1988).

${ }^{9}$ H.-S. Bosch et al., Nucl. Fus. 53, 126001(2013).

${ }^{10}$ T. Sunn Pedersen et al., Nucl. Fus. 55, 126001 (2015).

${ }^{11}$ R.C. Wolf et al., Nucl. Fus. 57, 102020 (2017).

${ }^{12}$ M. Krychowiak et al., Rev. Sci. Instrum. 87, 11D304 (2016).

${ }^{13}$ N. Krawczyk et al., Fus. Eng. Design 123, 727 (2017).

${ }^{14} \mathrm{M}$. Kubkowska et al., Fus. Eng. Design https://doi.org/10.1016/j.fusengdes.2017.12.024

${ }^{15}$ A. Czarnecka et al., Fus. Eng. Design https://doi.org/10.1016/j.fusengdes.2018.04.119

${ }^{16} \mathrm{M}$. Kubkowska et al., J. Instrum.10, P10016 (2015).

${ }^{17}$ http://amptek.com/products/mini-x-ray-tube/

${ }^{18} \mathrm{H}$. Thomsen et al., Journal of Instrumentation 10 (2015) P10015.

${ }^{19} \mathrm{~B}$. Buttenschön et al., Proceedings of 43rd EPS Conference on Controlled Fusion and Plasma Physics, Leuven, Belgium, 4-8 July 2016 (unpublished), Vol. 40A, P4.012.

${ }^{20}$ A. Langenberg et al. Fus. Sci. Techn.69, 560 (2016).

${ }^{21}$ A. Langenberg et al. Proceedings of 43rd EPS Conference on Controlled Fusion and Plasma Physics, Leuven, Belgium, 4-8 July 2016 (unpublished), Vol. 40A, P4.014.

${ }^{22} \mathrm{Th}$. Wegner et al., submitted to RSI (2018). 